\title{
CONTROLLED RELEASE IMPLANTS FOR CARDIOVASCULAR DISEASE*
}

\section{Robert J. Levy**}

The Division of Pediatric Cardiology, C.S. Mott Children's Hospital, and the Department of Pediatrics, The University of Michigan Medical School, and The College of Pharmacy of the University of Michigan, Ann Arbor, MI 48109 (U.S.A.)

\section{Thomas P. Johnston}

Department of Pharmaceutics, The College of Pharmacy, The University of Illinois at Chicago, Chicago, IL 606 12 (U.S.A.)

\section{Amnon Sintov}

Perio Products, P.O. Box 23950, Jerusalem (Israel)

\section{and Gershon Golomb}

Department of Pharmacy, School of Pharmacy, The Hebrew University of Jerusalem, P.O. Box 12065, Jerusalem 91120 (Israel)

Key words: controlled release implants; sustained release preparation; arrhythmia; calcification; pacemaker

The systemic therapy of many cardiovascular diseases is often hampered by adverse drug effects. The present paper examines the use of controlled release implants as a means for optimizing drug concentrations at the affected site in the cardiovascular system, while using a relatively low systemic dose. Controlled release systems have been prepared by combining a drug of choice with either a non-degradable polymer, such as a silicone rubber, polyurethane, and ethylene vinylacetate, or a biodegradable compound such as poly(glycolic-lactic acid) or a high molecular weight polyanhydride. Controlled release matrices containing ethylenehydroxydiphosphonate (EHDP), when implanted next to a bioprosthetic heart valve leaflet, prevented pathologic calcification. Similarly, controlled release matrices containing lidocaine-HCl have been used experimentally as epicardial implants to convert ventricular tachycardia to normal sinus rhythm in dogs. A matrix system containing gentamicin has been used by others [35] to prevent experimental valvular endocarditis. Other workers have used a dexamethasone-releasing cardiac pacing lead in clinical studies, to prevent scar tissue formation, which leads to elevated electrical pacing threshold [15,16]. Future controlled release systems for cardiovascular use will very likely incorporate innovative design features including: a reservoir configuration to replenish or change drug therapy, modulatable drug release to vary drug dosing as desired, and closed-loop feedback to increase or decrease release rates in response to disease status.

\footnotetext{
*Paper presented at the Fourth International Symposium on Recent Advances in Drug Delivery Systems, Salt Lake City, UT, U.S.A., February $21-24,1989$.

**To whom correspondence should be addressed at Robert J. Levy, M.D. Department of Pediatrics, The University of Michigan, R-5014 Kresge 11 0576, Ann Arbor, MI 481090576 (U.S.A.)
}

\section{INTRODUCTION}

The therapy of a variety of cardiovascular diseases is often hampered by adverse drug effects. For example, antiarrhythmic therapy using drugs such as propanolol, lidocaine, or 
amiodarone is often complicated by drug-related cardiac and systemic adverse effects [1$3]$. The use of controlled release implants is a potential way to avoid this type of problem and optimize treatment. The general strategy to be considered in this paper is that of implanting a drug delivery device at the site of an actual or potential pathologic process within the cardio vascular system. Local administration of a drug by this means is hypothesized to produce high regional concentrations, but relatively low net systemic doses. Thus, this approach would optimize therapy where it is needed and avoid high level drug exposure.

For the purpose of this paper controlled release will be defined as drug administration by means of an inert polymeric drug carrier. The drug may be incorporated into a controlled release system either as a dispersion in a monolithic matrix, or in the core of a reservoir. Matrices are typically formed with dispersions of uniform drug particles in biocompatible polymeric materials. Examples of nondegradable matrix polymers which have been widely used include: ethylene vinylacetate [4-6], silicone rubber [7-9], and polyurethane [10]. It is often advantageous to utilize biologically degradable matrix materials for controlled release implants, and this approach has been carried out using matrices composed of compounds such as poly (glycolic-lactic) acid [11], polyanhydrides [12], and poloxamers [13].

Reservoir configured controlled release systems have been less thoroughly investigated than matrices. However, reservoir systems offer a number of advantages including the ability to vary drug dosages, replenish drug supplies, or change therapy to a new pharmacologic agent. Controlled release reservoirs have been used as long-term (5 year) birth control implants [14], and this general strategy utilizing reservoirs will very likely be useful for cardiovascular disease as well.
Controlled release systems may be used to deliver a wide array of pharmacologic agents and macromolecules. This paper will describe in detail below the use of controlled release matrices for local administration of such diverse compounds as diphosphonates [5-7], steroids $[15,16]$, and antiarrhythmic compounds [10]. In addition, the work of Langer and Folkman [4], has demonstrated the potential for controlled release systems to deliver peptides and proteins over sustained periods ranging from weeks to months with intact biologic activity. Also of note, is the potential for varying the duration of controlled release depending upon the drug-polymer formulation [6,7] used and on the geometry utilized as well $[17,18]$. For example, hemispheric configured controlled release systems maintain a nearly constant drug release pattern while rectangular or cylindrical configured systems have a rather accelerated early phase of release followed by a continuous decline in drug release rates $[i 7,18]$. In addition, using drug salts of relatively lower solubility [7], as well as rate-limiting membranes [7], it is possible to sustain controlled release durations for periods of months to years. However, using either biodegradable systems or thin elastomeric films, release durations can also be made as brief as desirable.

Initial interest in the local administration of drugs in the cardiovascular system can be traced to the work of Mautz in the 1930s [19]. This investigator administered procaine into the pericardial sack of dogs and demonstrated a reduction in ventricular irritability. The pioneering of Folkman and Long [20] led to the first use of a controlled release system as a cardiac implant in the 1960s. These investigators fashioned silicone rubber reservoirs filled with various cardiac chronotropes. The silicone rubber reservoirs were demonstrated to function as pharmacologic pacemakers when they were loaded with agents such as thyroid hormone or norepinephrine and placed within the myocar- 
dium. Thus, these prior investigations demonstrated the potential for drug administration directly to the heart and were fundamental to subsequent discoveries.

\section{MATERIALS AND METHODS}

Poly (dimethyl siloxane)s (SIL) used for controlled release studies were medical grade Silastics (Dow Corning, Midland, MI). Ethylene vinylacetate was obtained from Dupont (Wilmington, DE), and the polyurethanes used in our studies were either Tecoflex $80 \mathrm{~A}$ or Thyomer (Thermedics, Woburn, MA). Ethanehydroxydiphosphonate (EHDP) as the disodium salt was provided by Norwich Eaton, Inc. (Norwich, $\mathrm{NY}$ ). Lidocaine $\mathrm{HCl}$ was provided by Abbott Laboratories (Chicago, IL). Other chemicals used were reagent grade as obtained from Sigma (st. Louis, MO). Bovine pericardium or porcine aortic valves were obtained fresh at slaughter and pretreated with glutaraldehyde as previously described [5-7]. Pretreated tissue was stored for at least 3 weeks or more prior to use.

Controlled release matrices were formulated as either drug dispersions in solid prepolymer [5-9] or as drug-polymer solutions in dimethylacetamide for certain polyurethane studies [10]. Matrices were cast in the desired geometric shape and were allowed to polymerize or solidify or both depending upon the formulation. In vitro release of controlled release matrices was carried out in an excess of a physiologic buffer (pH 7.4, $0.05 \mathrm{M}$ HEPES) at $37^{\circ} \mathrm{C}$ with frequent sampling of the releasing buffer for radiolabeled $\left({ }^{14} \mathrm{C}\right)$ drug $[5-10]$.

Rats used in the subdermal bioprosthetic implant calcification experiments were 3 week old male Sprague Dawley-CD (Charles River Inc., Burlington, MA) [5-7]. Sheep used in the open heart tricuspid valve replacement studies were male Suffolks, ages 5 to 7 months, weighing 40 to $50 \mathrm{~kg}$ at the time of surgery [8,9]. Bioprosthetic tissue explant assessment for calcification and morphology was carried out as previously described. The dogs used in the arrhythmia experiments were male mongrels ranging in weight from 10 to $20 \mathrm{~kg}$. The animals were monitored for electrocardiographic changes throughout these experiments [10], and periodic blood sampling for antiarrhythmic drug levels were obtained as well [10].

\section{RESULTS AND DISCUSSION}

\section{Preventing experimental cardiovascular calcification}

The formation of calcium phosphate deposits in blood vessels and heart valves is a common pathologic process complicating both primary cardiovascular disease and cardiovascular implant surgery [21]. There has been virtually no progress in the therapy of established cardiovascular calcifications, and prevention of the formation of these lesions has been complicated by side effects of the anticalcification agents. The bulk of the work in this area has focused on the use of diphosphonate compounds for prevention of experimental bioprosthetic heart valve calcification (Table 1).

Valvular bioprostheses are prepared with either glutaraldehyde-pretreated porcine aortic valves or stent-mounted glutaraldehyde-pretreated bovine pericardium [22]. These devices have been widely used as replacement cardiac valves because of their central orifice flow properties and relative nonthrombogenicity. However, calcification is the chief limiting factor leading to the clinical failure of these prostheses $[23,24]$. Intrinsic calcification occurs deep within the cupsal tissue and appears to be due to the propensity of the devitalized cells and structural protein matrix of glutaraldehydepretreated tissue to calcify. Excellent experimental models of this disease have been developed using either orthotopic valve replacements in large animals such as sheep [25] or calves [26], or subdermal implants in rats [26- 
TABLE 1

Inhibition of bioprosthetic heart valve leaflet calcification with controlled release ethanehydroxydiphosphonate (EHDP)

\begin{tabular}{|c|c|c|c|c|}
\hline Type of implant & Controlled release system & $n$ & $\begin{array}{l}\text { Explanted valve } \mathrm{Ca}^{2+} \\
(\mu \mathrm{g} / \mathrm{mg})\end{array}$ & $\operatorname{Ref}$ \\
\hline \multirow[t]{3}{*}{ Rat subdermal ${ }^{\mathrm{a}}$} & NaEHDP $(20 \%)$ in SIL & 10 & $2.3 \pm 0.5$ & 7 \\
\hline & NaEHDP: CaEHDP (20\%) in SIL & 10 & $4.2 \pm 0.9$ & 7 \\
\hline & Control (no drug) & 10 & $160.0 \pm 9.1$ & 7 \\
\hline Unimplanted (pericardial) & - & 7 & $1.2 \pm 0.1$ & 27 \\
\hline \multirow{2}{*}{$\begin{array}{l}\text { Shcep tricuspid } \\
\text { valve replacement }\end{array}$} & $\mathrm{NaEHDP}: \mathrm{CaEHDP}(30 \%)$ in SIL & 5 & $2.7 \pm 1.0$ & 8 \\
\hline & Control (no drug) & 6 & $41.3 \pm 14.9$ & 8 \\
\hline $\begin{array}{l}\text { Unimplanted } \\
\text { (porcine aortic valve) }\end{array}$ & - & 10 & $2.8 \pm 0.7$ & 28 \\
\hline
\end{tabular}

a 21 day implants using 3 week old male CD rats.

${ }^{\mathrm{b}} 150$ day implants using 5-7 months old male Suffolk sheep.

28]. In either case, the pathologic lesions produced have been shown to be comparable to those noted in clinico-pathologic specimens [26-28].

Systemic therapy using ethanehydroxydiphosphonate administered to animals with bioprosthetic tissue implants prevents bioprosthetic calcification $[29,30]$. The dose required to achieve this effect also results in skeletal diphosphonate toxicity and calcium imbalance $[29,30]$. However, controlled release administration of EHDP incorporated to either ethylene vinylacetate matrices [5,6] or silicone rubber matrices [7] resulted in the inhibition of bioprosthetic leaflet calcification at a dose of sufficiently low so as to avoid toxic side effects.

Controlled release diphosphonate has been demonstrated to be effective in the subdermal experimental model [5-7], although this approach has not been clearly beneficial in circulatory studies [8]. A notable aspect of this work has been the use of mixtures of the sodium and calcium salts of ethanehydroxydiphosphonate (EHDP) [7]. This has facilitated prolonging the release duration of the matrix so that a one to one mixture of sodium and calcium EHDP in a silicone rubber matrix as a $20 \%$ loading has an extrapolated release duration of more than
30 years [7,8]. A matrix composed of a $20 \%$ loading dicalcium EHDP in silicone rubber has an extrapolated release duration of more than 100 years and still maintains drug efficacy in the subdermal model [7].

The use of a controlled release matrix in conjugation with a functioning bioprosthetic heart valve is very challenging. It is necessary to position the controlled release matrix so that the drug can enter the heart valve leaflet tissue directly without being washed away by circulation. However, the controlled release matrix cannot interfere with the mechanical aspects of heart valve function. The strategy that has been used to surmount these difficulties has been to position the matrix as a periannular implant releasing drug to the site of heart valve leaflet attachments (Fig. 1). The diffusion of the drug would then take place from the attachment point into distal leaflets. One obvious limitation of this approach is the concentration gradient which must occur from the valve attachment points to the free edges, which results in less drug being present in the most distal aspects of the heart valve. A way around this is to preload heart valve leaflets with either a relatively insoluble diphosphonate salt such as calcium EHDP [31], or a covalently bound di- 


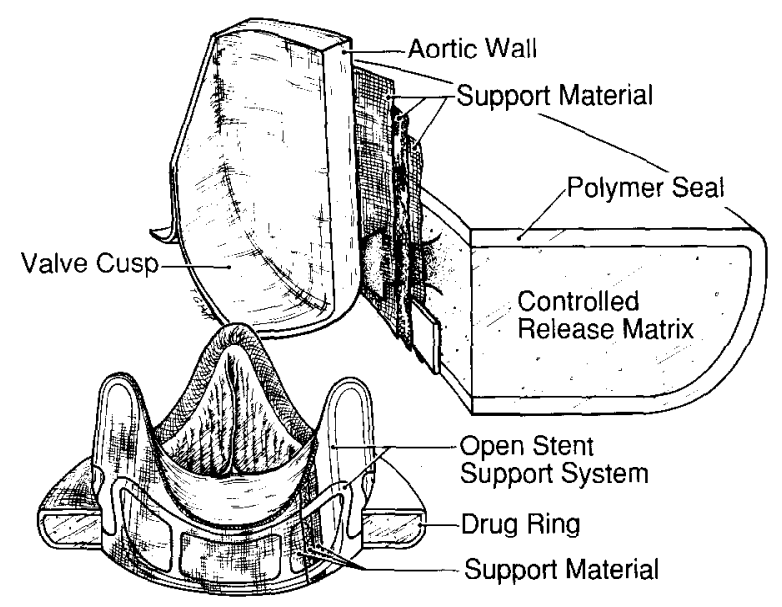

Fig. 1. An illustration of a controlled release matrix containing ethanehydroxydiphosphonate (EHDP) as a periannular sewing cushion in a porcine aortic bioprosthetic heart valve. Shown is sealing of the non-releasing surfaces to direct drug toward'the valve leaflets at their attachment points, with drug diffusion proceeding throughout the leaflets. Reproduced with permission from Replacement Cardiac Valves, edited by E. Bodner and R. Frater, Pergamon Press, Elsmford, NY, 1989.

phosphonate such as aminopropanehydroxydiphosphonate, which binds to residual aldehyde groups [32].

\section{Controlled release for cardiac arrhythmias}

Recent work from our laboratory has demonstrated the feasibility of controlled release administration of lidocaine from polyurethane matrices into the epicardium in dogs with experimental ventricular tachycardia (Fig. 2, Table 2) [10]. In our initial experiments ventricular tachycardia was induced with ouabain administration and a controlled release matrix was placed at a consistent location on the left ventricular epicardium [10]. Conversion of the ventricular tachycardia took place by $1.5 \mathrm{~min}$ and resulted in plasma lidocaine levels which roughly were one quarter of those noted with the identical drug doses administered intravenously. Lidocaine, in addition to having severe systemic side effects, is also a myocardial depressant. Therefore, in collaboration with Dr.
Kim Gallagher of the University of Michigan, the effect of epicardial lidocaine on myocardial thickening was assessed with implanted sonomicrometers [33,34]. Minimal (10\%) depression of ventricular thickening was noted with a dose of $7.5 \mathrm{mg} / \mathrm{kg}$ of lidocaine. However, when doses as low as $0.3 \mathrm{mg} / \mathrm{kg}$ were administered into the coronary circulation, profound depression of thickening (greater than $30 \%$ ) were noted. Thus, epicardial administration of lidocaine with a controlled release matrix takes place without adverse effects on cardiac function.

Subsequent investigations have also demonstrated the efficacy of epicardial lidocaine administration with the controlled release matrices for another model of experimental ventricular tachycardia, which was produced with rapid subthreshold impulses (cycle length of 50 $\mathrm{ms}$ ) [34] administered to epicardial ventricular electrodes in dogs. In this model system, controlled release lidocaine dosages on the order of $0.01 \mathrm{mg} / \mathrm{kg}$ were effective for conversion of ventricular tachycardia to sinus rhythm. It should be noted that these doses are approximately 100 times below conventional therapeutic dosages. Although initial investigations with this model system focused on lidocaine, subsequent studies have demonstrated efficacy of epicardial controlled release for a number of other antiarrhythmic agents including: procainamide, amiodarone, verapamil, flecainide, encainide, and nickel chloride. Ongoing work is investigating the use of these matrix systems for arrhythmias due to acute ischemia.

\section{Other uses of cardiovascular controlled release}

Cardiac controlled release is currently in clinical use with the Medtronic steroid eluting pacing catheter. This concept was based on the pioneering work of Stokes and his colleagues $[15,16]$. The use of cardiac pacemakers is frequently limited due to increases in the electrical pacing threshold caused by the formation of 


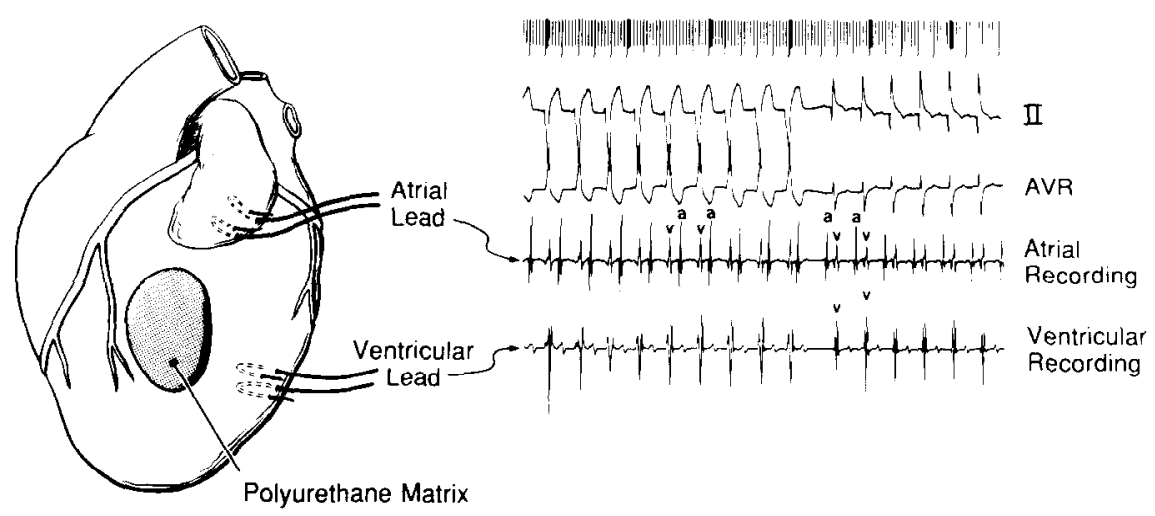

Fig. 2. An illustration of epicardial placement of a lidocaine $\mathrm{HCl}$ polyurethane controlled release matrix on a dog's heart with electrocardiographic conversion of ouabain induced ventricular tachycardia to normal sinus rhythm. Reproduced with permission from Elsevier Science Publishers (see Ref. 10).

TABLE 2

Controlled release lidocaine for the conversion of ventricular tachycardia in dogs

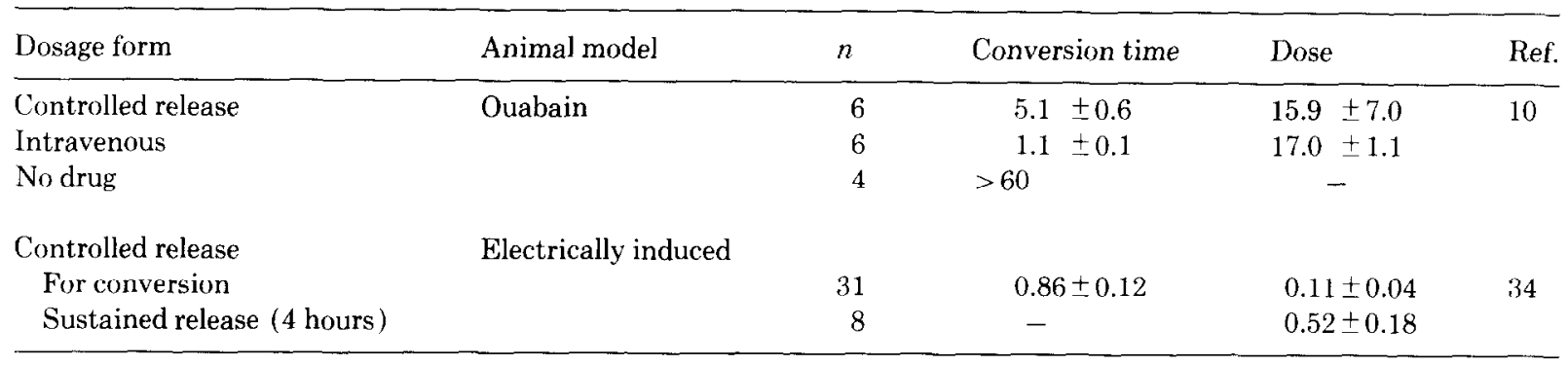

scar tissue at the pacing electrode tip. In order to counteract this phenomenon, Stokes and his colleagues formulated a silicone rubber controlled release system containing dexamethasone $[15,16]$. This antiinflammatory agent is slowly released over many years time from the pacing tip of the catheter into the endocardium in which the lead is embedded. Drug release at this site prevents scar tissue build-up and thus results in the maintenance of a relatively low electrical threshold for cardiac pacing. Dexamethasone is a potent antiinflammatory steroid, and thus the drug effect per dose administered is very much optimized. In addition, the site specificity of administration of dexamethasone has been maintained by administering the drug directly into the myocardium in a config- uration which avoids circulatory washout.

Another example of a different type of cardiovascular controlled release system is that of Olanof, Anderson, and Jones [35], who demonstrated successful controlled release of antibiotics to prevent prosthetic valvular endocarditis in a dog model. These investigators dispersed gentamicin into silicone rubber rings which were then incorporated in a periannular configuration surrounding the sewing cushion of a mechanical heart valve prostheses. In vitro drug release, and in vivo release and efficacy were documented for preventing bacteremia in this dog model. This particular approach would be most suitable for cardiac valve replacement in the setting of valvular bacterial endocarditis. 


\section{CRITICAL ASPECTS AND FUTURE CONSIDERATIONS}

An important limitation of controlled release matrices is their finite drug supply. Although it is possible for matrix release to be prolonged through through the use of rate-limiting membranes [7] and relatively insoluble drug salts $[7,8]$, this approach results in lower net drug dosages, which may not be effective for the particular disease of interest. Reservoir systems offer a number of advantages in this regard. Reservoir devices could be assessed percutaneously and the drug concentration may be altered as needed. Alternatively, the drug supply can be replenished or the therapeutic agent changed advantageously. Recent work from our laboratory has demonstrated the feasibility of this appraoch for a controlled release reservoir containing ethanehydroxydiphosphonate [36]. Polyurethane reservoirs containing a mixture of the sodium and calcium salt demonstrated sustained release properties with an extrapolated release duration of more than five years, and yet with anticalcification efficacy in the subdermal model [36].

Another important consideration is the fact that the controlled release systems described thus far in this paper have release rates which cannot be altered once the devices have been implanted. However, modulation of controlled release is possible through the use of either ultrasound [37] or electromagmetism [38]. In particular, electromagnetic modulation of controlled release is a particularly rapid and sensitive way of increasing or decreasing release rates as desirable. Langer and his colleagues have demonstrated that the incorporation of magnets into polymeric matrices permits modulation by an external electromagnetic field. The release rate, in fact, has been demonstrated to be significantly correlated with magnetic field strengths and the response time of their ethylene vinylacetate system was on the order of minutes [38]. Thus, for diseases such as cardiac arrhythmias where a rapid increase or decrease in drug delivery rate would be desirable, modulated systems such as the electromagnetic approach would be advantageous.

In addition, it would be most desirable for certain cardiovascular diseases to have closedloop feedback responsiveness regulating controlled release. The best example of a situation in which this would be desirable is that of cardiac arrhythmias. Cardiac arrhythmias can be readily sensed through computer-mediated algorithms which detect changes in the electrocardiographic patterns. Once these changes have been sensed a controlled release matrix, such as the electromagnetic one just described, could then be triggered and could provide increasing levels of drug until the arrhythmia had subsided. The computer technology needed to carry out the sensing and triggering aspects of this approach already exists and is being used currently in the implantable defibrillator systems [39]. What remains to be done is for a suitable controlled release formulation to be coupled with this existing technology.

There are other potential future uses for controlled release for cardiovascular disease. It may be advantageous to administer immunosuppressives following cardiac transplantation directly into the heart to avoid such complications as cyclosporin-mediated hypertension [40] and encephalopathy [41]. Controlled release matrices might also be advantageous for thrombolysis and anticoagulation, if used as intravascular implants. Further, controlled release intravascular implants containing free radical scavenging enzymes and related agents may also one day be therapeutically useful in the therapy of ischemic organ damage [42].

\section{CONCLUSION}

Controlled release implants have been demonstrated to be effective for a number of settings related to cardiovascular disease. Dexamethasone-silicone rubber matrices for preventing excessive scar tissue formation are 
already in clinical use in a cardiac pacing catheter configuration. Other successful experimental uses of controlled release include: prevention of cardiovascular calcification with diphosphonates; therapy of ventricular tachycardia with lidocaine $\mathrm{HCl}$ and other antiarrhythmic agents; and prevention of cardiac valve prosthesis endocarditis with an antibiotic eluting valve prosthesis sewing cushion. In each instance, local drug concentrations are optimally elevated, while systemic drug exposure is relatively low, limiting the possibilities for adverse drug effects.

\section{ACKNOWLEDGMENT}

This work was supported in part by NIH Grants HL38118 and HL36574. Dr. Johnston was a Fellow of the American Heart Association of Michigan and Dr. Levy is an Established Investigator of the American Heart Association. We thank Mrs. Catherine Wongstrom for her expertise in preparing this manuscript and we also thank Mrs. Maria Lehto and Mrs. Kathleen Reekel for their expert technical assistance. We also thank our illustrators, Ted Huff (Fig. 1) and Chris Burke (Fig. 2) of the Biomedical Communications Dept. of the University of Michigan Medical School. We are also grateful to both Dr. William Pfister of Dow Corning for his advice concerning the use of Silastic, and to Dr. Kent Buckingham of Norwich Eaton for providing EHDP.

\section{REFERENCES}

1 S.S. Sheu and W.J. Lederer, Lidocaine's negative inotropic antiarrhythmic actions. Dependence on shortening of action potential duration and reduction of in tracellular sodium activity, Circ. Res., 57 (1985) 578 590.

2 A.A. De Paola, L.N. Horowitz, S.K. Spielman, P. Brady, J. Morganroth, A.M. Greenspan and H.R. Kay, Development of congestive heart failure and alterations in left ventricular function in patients with sustained ventricular tachyarrhythmias treated with amiodarone, Amer. J. Cardiol., 60 (1987) 276280.
3 F. Morady, W.H. Kou, S.D. Nelson, M. De Buitleir, S. Schmaltz, A.H. Kadish, L.K. Toivonen and J.A. Kushner, Accentuated antagonism between beta-adrenergic and vagal effects on ventricular refractoriness in humans, Circulation, 77 (1988) 289-297.

4 R. Langer and J. Folkman, Polymers for the sustained release of proteins and other macromolecules, Nature, 263 (1976) 797-800.

5 R.J. Levy, J. Wolfrum, F.J. Schoen, M.A. Hawley, S. Lund and R. Langer, Inhibition of calcification of bioprosthetic heart valve calcification by local controlled release of diphosphonate, Science, 228 (1985) 190-192.

6 G. Golomb, R. Langer, F.J. Schoen, M.S. Smith, Y.M. Choi and R.J. Levy, Controlled release of diphosphonate to inhibit bioprosthetic heart valve calcification: dose-response and mechanistic studies, J. Controlled Release, 4 (1986) 181-194.

7 G. Golomb, M. Dixon, M.S. Smith, F.J. Schoen and R.J. Levy, Controlled release drug delivery of diphosphonate to inhibit bioprosthetic heart valve calcification: release rate modulation with silicone matrices via drug solubility and membrane coating, J. Pharm. Sci., 76 (1987) 271-276.

8 T.P. Johnston, E.L. Bove, S.F. Bolling, R.J. Schoen, J.A. Boyd, G. Golomb and R.J. Levy, Local controlled release of 1-hydroxyethylidene diphosphonate using silicone rubber matrices, Trans. Amer. Soc. Artif. Intern. Organs, 34 (1988) 835-838.

9 T.P. Johnston, E.L. Bove, S.F. Bolling, J.A. Boyd, B.L. Ciesliga, G.L. Amidon, F.J. Schoen and R.J. Levy, Controlled release of l-hydroxyethylidene diphosphonate: in vitro assessment and effects on bioprosthetic calcification in sheep tricuspid valve replacements, Int. J. Pharm., 52 (1989) 139-148.

10 A. Sintov, W. Scott, M. Dick and R.J. Levy, Cardiac controlled release for arrhythmia therapy: lidocainepolyurethane matrix studies, J. Controlled Release, 8 (1988) 157-165.

11 J.P. Kitschell and D.L. Wise, Polylactic/glycolic biodegradable drug-polymer matrix system, Meth. Enzymol., 112 (1985) 436-448.

12 A. Domb and R. Langer, Preparation of high molecular weight polyanhydrides, J. Polym. Sci., 25 (1987) 3373-3386.

13 T.P. Johnston and S.C. Miller, Toxicological evaluation of poloxamer vehicles for intramuscular use, J. Parenter. Sci. Technol., 39 (1985) 83-89.

14 Y.W. Chien, Microsealed drug delivery systems: fabrication and performance, Meth. Enzymol., 112 (1985) 461-470.

15 R. Langer and N. Peppas, Chemical and physical structure of polymers as carriers for controlled release of bioactive agents: a review, Macromol. Chem. Phys., 23 (1983) 61-126. 
16 H. Mond, K. Stokes, J. Helland, L. Grigg, P. Kertes, B. Pate and D. Hunt, The porous titanium steroid eluting electrode: a double blind study assessing the stimulation threshold effects of steroid, PACE, 11 (1988) 214-219.

17 A.S. Radovsky, J.F. Van Vleet, K.B. Stokes and W.A. Tacker, Paired comparisons of steroid eluting and nonsteroid endocardial pacemaker leads in dogs: electrical performance and morphologic alterations, PACE, 11 (1988) 1085-1094.

18 D.S.T. Hsieh, W.D. Rhine and R. Langer, Zero order controlled release polymer matrices for micro and macromolecules, J. Pharm. Sci., 72 (1983) 17-22.

19 F.R. Mautz, Reduction of cardiac irritability of the epicardial and systemic administration of drugs as a protection in cardiac surgery, J. Thorac. Surg., 5 (1936) 612-619.

20 J. Folkman and D.M. Long, The use of silicone rubber as a carrier for prolonged drug therapy, J. Surg. Res., 4 (1964) 139-149.

21 F.J. Schoen, H. Harasaki, K.M. Kim, H.C. Anderson and R.J. Levy, Biomaterials associated calcification: pathology, mechanisms, and strategies for prevention, J. Biomed. Mater. Res., Appl. Biomater., 22 (A1) (1988) 11-36.

22 F.J. Schoen, J.L. Kujovich, R.J. Levy and M.S.J. Sutton, Bioprosthetic valve failure, in: B.F. Waller (Ed.), Cardiovascular Clinies, F.A. Davis, Philadelphia, PA, 1987, pp. 289-318.

23 A. Milano, U. Burtolotti, E. Talenti, C. Valfre, E. Arbustini, M. Valente, A. Mazzucco, V. Gallucci and G. Thiene, Calcific degeneration as the main cause of porcine bioprosthetic valve failure, Amer. J. Cardiol., 53 (1984) 1066-1070.

24 F.J. Schoen, J.L. Kujovich, C.L. Webb and R.J. Levy, Chemically determined mineral content of explanted porcine aortic valve bioprostheses: correlation with radiographic assessment of calcification and clinical data, Circulation, 76 (1987) 1061-1066.

25 E. Arbustini, M. Jones, R.D. Moses, E.E. Eidbo, R.J. Carroll and V.J. Ferrans, Modification by the Hancock T6 process of calcification of bioprosthetic cardiac valves implanted in sheep. Amer. J. Cardiol., 53 (1984) 1388-1396.

26 F.J. Schoen, R.J. Levy, A.C. Nelson, W.F. Bernhard, A. Nashef and M. Hawley, Onset and progression of experimental bioprosthetic heart valve calcification, Lab. Invest., 52 (1985) 523-532.

27 R.J. Levy, R.J. Sehoen, J.T. Levy, A.C. Nelson, S.L. Howard and L.J. Oshry, Biologic determinants of dystrophic calcification and osteocalcin deposition in glutaraldehyde preserved porcine aortic valve leaflets implanted subcutaneously in rats, Amer. J. Pathol., 113 (1983) 143-155.
28 F.J. Schoen, J. Tsao and R.J. Levy, Calcification of bovine pericardium used in cardiar valve binprostheses: role of glutaraldehyde-modified structural components in bioprosthetic tissue mineralization, Amer. J. Pathol., 123 (1986) 134-135.

29 R.J. Levy, M.A. Hawley, F.J. Schoen, S.A. Lund and P.Y. Liu, Diphosphonate compounds inhibit calcification of porcine bioprosthetic heart valve cusps implanted subcutaneously in rats, Circulation, 71 (1985) 349356.

30 R.J. Levy, F.J. Schoen, S.A. Lund and M.S. Smith, Prevention of leaflet calcification of bioprosthetic heart valves with diphosphonate injection therapy: experimental studies of optimal dosages and therapeutic durations, J. Thorac. Cardiovase. Surg., 94 (1987) 551557.

31 T.P. Johnston, F.J. Schoen and R.J. Levy, Prevention of calcification of bioprosthetic heart valve leaflets by $\mathrm{Ca}^{++}$diphosphonate pretreatment, J. Pharm. Sci., 77 (1988) 740-744.

32 C.L. Webb, J.J. Benedict, F.J. Schoen, J.A. Linden and R.J. Levy, Inhibition of bioprosthetic heart valve calcification with aminodiphosphonate covalently bound to residual aldehyde groups, Ann. Thorac. Surg., 10 (1987) 592-595.

33 W.A. Scott, A. Sintov and R.J. Levy, Polymer matrices for myocardial lidocaine delivery, Ped. Res., 23 (1988) 225A.

34 A. Sintov, R. Siden and R.J. Levy, Myocardial controlled release lidocaine for ventricular tachycardia in dogs, Pharm. Res., 5 (1988) S-64.

35 L.S. Olanof, J.M. Anderson and R.D. Jones, Sustained release of gentamicin from prosthetic heart valves, Trans. Amer. Soc. Artif. Intern. Organs, 25 (1979) 334-338.

36 T.P. Johnston, J.A. Boyd, B.L. Ciesliga, F.J. Schoen and R.J. Levy, Controlled release of 1-hydroxyethylidene diphosphonate from polyurethane reservoirs to inhibit calcification of bioprosthetic heart valves, $\mathrm{Cir}$ culation, 78 (1988) II-596.

37 J. Kost, D. Levy and R. Langer, Ultrasound effect on transdermal drug delivery, Proceedings of the 13th International Symposium on Controlled Release, 1986, pp. 171-178.

38 E.R. Edelman, L. Brown, J. Taylor and R. Langer, In vitro and in vivo kinetics of regulated drug release from polymer matrices by oscillating magnetic fields, $J$. Biomed. Mater. Res, 21 (1987) 339-353.

39 J. Jenkins, T. Bump, F. Munkenbeck, J. Brown and R. Arzbaecher, Tachycardia detection in implantable antitachycardia devices, PACE, 7 (1984) 1273-1277.

40 P.C. de Groen, A.J. Askamit, J. Rakela, G.S. Forbes and R.A. Krom, Central nervous system toxicity after liver transplantation. The role of cyclosporine and cholesterol, N. Engl. J. Med., 317 (1987) 361-866. 
41 M.L. Greenberg, B.F. Uretsky, P.S. Reddy, R.L. Bernstein, B.P. Griffith, R.L. Hardesty, M.E. Thompson and H.T. Bashon, Long-term hemodynamic follow-up of cardiac transplant patients treated with cyclosporine and prednisone, Circulation, 71 (1985) 487-494.
42 R. Bolli, B.S. Patel, M.O. Jeroudi, E.K. Lai and P.B. McCay, Demonstration of free radical generation in "stunned" myocardium of intact dogs with the use of the spin trap alpha-pentyl $N$-tert-butyl nitrone, J. Clin. Invest., 82 (1988) 476-485. 